\title{
IdeAs
}

Idées d'Amériques

\section{Des lieux avec lesquels penser, des livres auxquels penser}

Mots, expérience et décolonisation de la connaissance dans les Andes boliviennes

Places to think with, books to think about

Lugares para pensar con ellos, Libros para pensar en ellos

\section{Anders Burman}

Traducteur : Laura Pertuy

\section{OpenEdition}

\section{Journals}

Édition électronique

URL : https://journals.openedition.org/ideas/238

DOI : $10.4000 /$ ideas.238

ISSN : 1950-5701

Éditeur

Institut des Amériques

Référence électronique

Anders Burman, "Des lieux avec lesquels penser, des livres auxquels penser », IdeAs [En ligne], 2 I

2012, mis en ligne le 22 juin 2012, consulté le 19 octobre 2022. URL : http://journals.openedition.org/ ideas/238; DOl : https://doi.org/10.4000/ideas.238

Ce document a été généré automatiquement le 19 octobre 2022

\section{cc)}

Creative Commons - Attribution - Pas d'Utilisation Commerciale - Pas de Modification 4.0 International - CC BY-NC-ND 4.0

https://creativecommons.org/licenses/by-nc-nd/4.0/ 


\section{Des lieux avec lesquels penser, des livres auxquels penser}

Mots, expérience et décolonisation de la connaissance dans les Andes boliviennes

Places to think with, books to think about

Lugares para pensar con ellos, Libros para pensar en ellos

\section{Anders Burman}

Traduction : Laura Pertuy

J'aimerais remercier Carlos Yujra pour ses enseignements ainsi que Ramón Grosfoguel et les autres participants de la conférence "Quels universités et universalismes demain en Europe ? Un dialogue avec les Amériques » qui a eu lieu à Paris, en juin 2010, pour leurs commentaires sur la première version de cet essai.

1 Dans la Bolivie d'aujourd'hui, les combats politiques des peuples indigènes se mêlent à des problèmes de connaissance et de vérité. Les cosmologies indigènes et les systèmes «traditionnels» de connaissance ne sont pas seulement politisés comme jamais auparavant, mais ils permettent également une lecture de la réalité et du fonctionnement politique national qui diffère des opinions dominantes et les affaiblit.

Afin d'introduire le sujet de cet essai - l'épistémologie aymara - j'aimerais citer quelqu'un que l'on évoque très peu lors des discussions au sujet des peuples indigènes et de la décolonisation de la connaissance : le botaniste suédois du XVIII ${ }^{e}$ siècle Carl Linnaeus. Je le mentionne non pas pour impressionner le lecteur avec des références rares mais justement pour apporter une nuance à l'épistémologie moderne et coloniale de la raison scientifique de Linnaeus (voir ex. Jensen 2009) grâce à une épistémologie indigène subalternisée. Dans son Systema Naturae (1735), Linnaeus identifiait la race humaine comme une espèce particulière au sein du royaume animal. Il rencontra cependant quelques problèmes à apporter un argument solide dans la différenciation de l'anatomie humaine de celle des singes. Il proposa comme critère ultime la faculté intellectuelle de la raison qui distingue les humains des singes. Seuls les humains, selon Linnaeus, ont les moyens rationnels et systématiques de produire et de transmettre une 
véritable connaissance $\mathrm{du}$ monde et d'eux-mêmes. Ou, comme Tim Ingold l'a strictement formulé : «Il n'y a guère de scientifiques chez les animaux » (2000:238).

3 En 2010, un ami, jeune intellectuel et activiste indigène d'origine bolivienne aymara, que j'appellerai ici Antonio, a également établi la distinction entre humains et nonhumains au regard de la sphère de la connaissance mais en se basant sur des fondements radicalement différents de ceux de Linnaeus. Selon Antonio, la connaissance transmise par le langage n'est que pur «siwsawi», c'est-à-dire de la discussion, des opinions, des points de vue et jugements émanant de certains individus. Comme telle, il s'agit d'une connaissance d'un type particulier, une connaissance concernant les opinions d'autres êtres humains et rien d'autre. Celle-ci se distingue ainsi grandement de la connaissance non-linguistique et expérientielle qui prend racine dans le vécu, qui est obtenue dans, avec, à partir du monde et en son sein, avec et à partir des plantes, des montagnes, des lacs, des animaux sans oublier certains lieux de sagesse présents dans le paysage et appelés wak'as. Ce type de connaissance, selon mon ami, se nomme "ukanaw », autrement dit les choses telles qu'elles sont : si les humains mentent parfois, ce n'est pas le cas de certains autres sujets savants et constitutifs de ce monde (à l'exception de quelques " animaux farceurs »). Même si Antonio rejoindrait certainement Linnaeus sur le fait qu'il n'existe pas de scientifiques parmi les animaux, il affirme résolument qu'il n'y a aucun charlatan parmi les plantes, ni aucun menteur parmi les montagnes.

Martha Hardman (1986) a montré que les personnes de langue aymara utilisent constamment des «marqueurs de source de l'information » (généralement en ajoutant des suffixes à leurs phrases) afin d'indiquer s'ils puisent leurs sources dans des connaissances personnelles expérientielles, dans des connaissances acquises par le langage ou bien dans des connaissances non-personnelles. Cet essai se penchera en détails sur les deux premières catégories. Quand Antonio fait la distinction entre la nature "siwsawi » de la connaisance acquise via le langage humain et la nature "ukanaw" de la connaissance personnelle acquise via l'interaction non-linguistique avec d'autres sujets sages présents dans le monde, il utilise, selon Hardman, une logique linguistique aymara qui est "si omniprésente que les locuteurs considèrent le problème comme intégré à la nature de l'univers (1986:114). Ainsi, le fait de ne pas préciser à quel type de connaissance on se réfère est considéré avec méfiance : «Ceux qui arrivent dans la communauté depuis l'extérieur et avancent des faits comme connaissances personnelles alors qu'ils ne les connaissent que via le langage (par exemple les choses qu'ils ont lues dans les livres) sont immédiatement taxés de rustres » (Hardman 1986 :133).

Cette façon de faire la distinction entre différents types de connaissances en se basant sur leur source et leur supposée fiabilité présente des implications intéressantes dans l'actuel processus de « décolonisation » de l'université bolivienne et la création récente «d'universités indigènes » comme parties intégrantes de la politique d'état visant la " décolonisation » et lancée par l'administration d'Evo Morales en 2006. Cependant, la signification de "décolonisation" est loin d'être limpide pour la société bolivienne contemporaine. Lors de discours officiels, le gouvernement a fait preuve d'une capacité remarquable à étendre de manière sémantique et politique le concept de "décolonisation ». Par exemple, ce dernier peut correspondre à "développement ", « industrialisation», «modernisation", «patriotisme», «nationalisation » et "croissance économique ", mais il peut aussi dénoter une franche critique assortie de 
mesures politiques contre l'impérialisme, le capitalisme, le néolibéralisme, le racisme, le sexisme, le développementarisme, la déprédation écologique et, dans le secteur de la production de la connaissance et de l'éducation, l'eurocentrisme, la surestimation de n'importe quelle tradition de pensée venant du nord ainsi que l'infériorisation concomitante de n'importe quelle tradition indigène (voir Burman 2011 :69).

6 Antonio est né et a été élevé dans une communauté rurale aymara. Ceci dit, il vit actuellement plus ou moins de manière permanente dans la ville d'El Alto et il étudie les sciences politiques à l'université de La Paz. Bien qu'il soit loin d'être le seul étudiant de langue maternelle aymara, les cours sont uniquement dispensés en espagnol. Même si l'espagnol parlé dans les Andes boliviennes a été influencé de plusieurs façons par les langues andines et que la production de références est une pratique académique établie, il n'existe pas véritablement d'emploi des «marqueurs des sources de l'information » chez les professeurs. Ainsi, alors que le professeur, généralement un homme non-indigène (bien que cela soit en train de changer), pense qu'il donne un cours sur les choses telles qu'elles sont, Antonio et ses camarades aymara émettent quelques doutes. Lorsque le professeur parle de la façon dont Karl Marx a écrit Das Kapital à Londres sans mentionner qu'il tient ces informations de connaissances non-personnelles acquises via la langue humaine (par exemple par l'intermédiaire d'un livre), il leur semble qu'il parle en s'appuyant sur des connaissances personnelles expérientielles comme s'il avait été au bureau de Marx à Londres et avait fait l'expérience de l'écriture du livre. Puisqu'Antonio et ses camarades aymara savent très bien, grâce aux faits établis, que Marx a publié le premier volume de son magnus opus dans les années 1860, le professeur - comme il n'a pas formulé de «marqueurs de sources de l'information " corrects - leur semble être un orateur se réclamant d'une connaissance bien trop grande par rapport à la réalité. Ce n'est pas comme si Antonio et ses amis pensaient vraiment que le professeur prétendait avoir été présent dans les années 1860 à Londres; cependant, selon eux, celui-ci fait preuve d'une façon confuse de gérer différentes sortes de connaissances puisqu'il méprise les implications du fait que ce qu'il déclare comme vrai prend racine dans différents types de sources et que les différentes revendications de connaissances ont ainsi différents degrés de fiabilité. De sorte que, quand différents professeurs présentent des affirmations confuses et injustifiées, Antonio en vient à se dire que les conférences et les livres sont bien utiles si l'on est intéressé par l'opinion des gens, leurs idées et jugements, mais qu'ils ne sont rien d'autre que du «siwsasi»: des mots dits, entendus et lus, non enracinés dans l'expérience.

7 Je pose ici une question: si les livres et conférences ne concernent que les opinions d'individus spécifiques et que la vraie connaissance s'acquière dans les échanges expérientiels, non-linguistiques et inter-relationnels avec le monde et en son sein, n'ya-t-il pas un risque qu'un projet destiné à décoloniser la connaissance et l'université grâce aux livres et aux conférences, c'est-à-dire un projet de décolonisation logocentrique ou comme je le suggérerais «librocentrique ", finisse par reproduire les asymétries épistémologiques coloniales de production de la connaissance ? Cet essai, d'une part, examine méticuleusement les problèmes liés à la nature «siwsawi» de la connaissance académique conventionnelle vis-à-vis d'un processus de décolonisation critique et créatif; d'autre part, il explore la nature "ukamaw» de la connaissance expérientielle et les possibilités, via ce type de connaissance, de poser les fondations d'une transformation épistémologique décoloniale de l'université bolivienne et des universités indigènes récemment établies. L'essai pose foncièrement la question du 
sens de " savoir ", de ce qu'est la connaissance, et de ce que signifie être un sujet savant et instruit dans les Andes boliviennes aujourd'hui. C'est -à-dire dans un contexte où les traditions de pensée subalternisées manifestent de nouveaux besoins urgents en termes de dynamiques éducatives et politiques et au sein desquelles différents points de vue et revendications de vérité coexistent, se mélangent et entrent en collision.

\section{L'épistémologie et la colonialité de la connaissance}

Dans ses commentaires sur l'article "L'animisme revisité » de Nurit Bird-David, Eduardo Viveiros de Castro (1999:79) avance que les peuples indigènes n'ont consacré aucun effort intellectuel notable pour faire disparaître cette question essentiellement moderne qu'est l'épistémologie. Leur épistémologie appartient à la catégorie de l'ontologie selon Viveiros de Castro, et non à celle de l'épistémologie. Si l'on comprend épistémologie comme une discussion universitaire détachée, décontextualisée et désancrée sur la nature de la connaissance en tant que telle, il a certainement raison; dans ce sens-là, cela n'a jamais été un problème de grande importance pour les peuples indigènes en général. Cependant, comme Bird-David (1999 : 87) l'avance, «la modernité n'a aucun monopole sur de telles questions ", c'est-à-dire sur des questions telles que : «combien en savons-nous sur ce que nous savons?» et «qu'est-ce que la connaissance? ».

9 Il y a un homme qui formule de telles questions dans une perspective qui s'éloigne beaucoup des conventions modernes. Il s'agit de don Carlos Yujra Mamani, un chaman ${ }^{1}$ aymara approchant les 60 ans avec qui je dialogue sur ces questions depuis des années. Grâce à sa posture de chaman mais aussi d'activiste indianiste-katariste influent et radical, don Carlos incarne le mouvement actuel d'activisme indigène et de politiques d'état alliés à la cosmologie indigène et à la connaissance thérapeutique rituelle dans la société bolivienne (Burman ${ }^{2}$ 2010). Don Carlos ne connaît pas le mot "épistémologie " et bien qu'il soit toujours ravi d'ajouter de nouveaux termes "étrangers" à son espagnol fortement marqué par l'accent aymara, je doute fort qu'il aurait utilisé ce terme même s'il l'avait connu. Pour don Carlos, les problèmes pouvant être identifiés comme « épistémologiques » sont intéressants non pas parce qu'ils appartiennent à un type bien spécifique dont on pourrait parler avec détachement mais parce qu'ils ne peuvent être séparés des questions de santé, de politique, d'éthique et de vie tout court. Ils sont donc d'importance. De plus, dans les Andes, à cause de la prédominance "d'étranges " systèmes de connaissance depuis 500 ans - je fais ici référence à un fonctionnement colonial que le philosophe bolivien quechua Victor Hugo Quintanilla appelle « négation (...) des cultures indigènes et mise en place d'une culture d'un seul monde, d'un seul centre » (2009:128, ma traduction) - les problèmes épistémologiques sont devenus urgents, particulièrement pendant les décennies des grandes mobilisations indigènes. Quand on méprise l'ontologie, la cosmologie et jusqu'à l'existence d'un individu, les questions épistémologiques gagnent de l'importance. Car si des autres dominants et puissants proclament que la connaissance de la réalité d'un individu est fausse, alors la façon d'appréhender la réalité de cette personne devient erronée et sa façon de révéler la nature du monde trompeuse. Ainsi, défendre une ontologie, c'est défendre une épistémologie ; on revendique, on défend et on se réclame de la façon dont on sait; alors "comment sait-on ce que l'on sait?" devient une question d'importance politique, existentielle et même cosmologique. Le colonialisme 
produit des réactions anti-coloniales : de la résistance et de l'insurrection politique, cosmologique, ontologique et, par extension, épistémologique. Ainsi, pour vraiment comprendre pourquoi l'épistémologie s'est transformée en question d'importance pour les peuples indigènes des Andes, nous explorerons ce qui a été étiqueté « colonialité de la connaissance » (voir ex. Lander 2000 ; Walsh et. Al. 2002 ; Mignolo 2005).

Parler de colonialité de la connaissance, c'est reconnaître que la domination coloniale détient une dimension épistémique et que la violence épistémique est partie intégrante des relations coloniales de puissance qui caractérisent le monde depuis 1492 (voir, ex. Dussel 2008). Un des résultats de ce déséquilibre épistémologique s'incarne dans le fait que la connaissance de don Carlos, le chaman et activiste aymara introduit plus haut, est sans cesse infériorisée par rapport à celle d'un scientifique travaillant avec les pratiques discursives et les présupposés cosmologiques du Nord dominant. Le monde académique et l'université sont, sans conteste, des composants essentiels du système qui a établi l'asymétrie épistémologique et qui s'assure désormais de sa continuité dans le Nord mondialisé comme dans le Sud. Ainsi, Boaventura de Sousa Santos (2008) avance que le Nord - inscrit dans un processus de prédominance épistémique coloniale et producteur actif d'une absence épistémique où l'altérité épistémique est détectée - a diminué et continue de diminuer la diversité d'expériences et de systèmes de connaissances dans le monde et ainsi son potentiel à réinventer des alternatives à l'épistémologie hégémonique dominante du Nord. Il n'a pas seulement été question d'imposer une épistémologie dominante sur les subalternes coloniaux afin de remplacer les systèmes « locaux » de connaissance. Un enjeu tout aussi important de la machinerie coloniale de contrôle et de pouvoir a aussi été d'appréhender « l'Autre » (le Sud) sous le prisme des concepts hégémoniques et au sein de la logique dominante du «Soi » (le Nord) (Santos $2008: 30$ ). La pratique de l'anthropologie dans son entier a été lancée avec cette mission en tête. En d'autres termes, et comme mentionné par Edward Said (1978) et bien d'autres, la connaissance n'est jamais innocente et sa production n'est jamais déconnectée des mécaniques du pouvoir.

11 Tout comme il pourrait être problématique de parler d'une tradition de pensée aymara unique, il serait également problématique de parler d'une culture homogène de la connaissance unique dans la sphère que nous appelons le Nord, non seulement parce que le Nord n'est en aucun cas restreint à l'Europe et à l'Amérique du Nord, mais également car toutes les sociétés humaines font preuve d'hétérogénéité et de contradictions internes. En d'autres termes, il n'existe pas d' "épistémologie du Nord » homogène et consistante ou d'" épistémologie indigène " monolithique mais une multitude de courants épistémologiques qui se chevauchent, se confondent et se contredisent. Cependant, une insistance sur la nature fragmentaire du monde peut nous conduire à survoler la structure asymétrique du pouvoir qui l'étaye. Le monde souffre clairement d'un déséquilibre épistémologique. La connaissance produite au sein d'une certaine tradition de pensée, c'est-à-dire la tradition scientifique moderne du Nord, est considérée comme supérieure. Et qu'importe combien cette tradition peut être hétérogène, identifier certaines de ses caractéristiques est loin d'être infaisable.

L'épistémologie qui a été instituée dans le monde comme théorie hégémonique de la connaissance revendique la séparation du « connu » de « celui qui connaît » et du « fait de savoir ». Cette épistémologie prend en grande partie racine dans la métaphysique cartésienne. Selon Tim Ingold, il s'agit d'une épistémologie qui suppose le « désengagement total du sujet par rapport au monde » $2000: 169)$ et qui « injecte une 
séparation entre l'esprit et le monde, ou entre la raison et la nature, tel un à priori ontologique » (2000: 391). Ainsi, le sujet "qui sait» est renfermé sur lui-même et observe un monde d'objets puis produit une supposée connaissance de ces objets. Le sujet «qui sait» est ainsi capable de connaître le monde sans en faire partie et d'entretenir, dans tous les cas, un point de vue désintéressé par rapport à son sujet comme de produire une connaissance supposément universelle et indépendante de tout contexte. Cette notion hégémonique de production de la connaissance génère des pratiques scientifiques discursives et installe des cadres d'interprétation qui rendent difficile le fait de penser en dehors de ces mêmes cadres ; simultanément, cela réprime tout ce qui est articulé, pensé et envisagé en dehors de ces cadres.

\section{Des lieux avec lesquels penser}

13 Un an après l'accession au pouvoir d'Evo Morales, le chaman et activiste don Carlos se mit à travailler sur un projet interculturel lié à la santé - alors mené par le Ministère de la santé bolivien - avec pour tâche l'intégration d'une "connaissance indigène thérapeutique » dans le système médical national. Seuls quelques uns de ses collègues le comprenaient, selon ses dires. La plupart, pas du tout. « Ils pensent seulement avec la piqi (la tête), c'est parce que ce sont des imbéciles ", déclare-t-il. « Comment peuvent-ils possiblement savoir quoi que ce soit s'ils ne l'ont jamais ressenti? » se demande-t-il, soulignant la connexion intime entre « raison» et «ressenti » dans les concepts de connaissance aymara. Dans la plupart des études ethnographiques de conceptualisation corps-et-esprit chez les aymara, "raison » et "pensée » sont reliées à la piqi, c'est-àdire à la tête, alors que le ressenti et «l'émotion" sont reliées à la chuyma. La chuyma est souvent traduite par "cœur " étant donné que les qualités et les émotions s'y rapportant ressemblent à ce que les Boliviens disent s'installer dans le cœur. Cependant, le «cœur » dans un sens moins métaphorique est appellé Iluqu en Aymara et une traduction plus précise de chuyma serait en fait "poumons». Dans certaines occasions, la chuyma est désignée comme un organe spécifique avec des qualités particulières. En d'autres occasions, sa signification est étendue pour désigner non seulement les poumons mais aussi le thorax tout entier qui, comme l'a noté Andrew Orta (1999: 865), est le «siège somatique de la personne». Cependant, et comme ce sera exposé plus précisément plus tard, il y a aussi des circonstances où la chuyma désigne quelque chose de bien plus grand que le thorax et d'autres occasions où la subjectivité humaine n'est pas limitée à la peau humaine. Comme je le discute autre part (Burman 2010), toute distinction précise entre la faculté de raison supposément située dans la piqi et la faculté d'émotions supposément située dans la chuyma fausserait toute compréhension de l'épistémologie aymara et des conceptions du corps et de l'esprit. On pense aussi bien avec la chuyma qu'avec la piqi ou même plus ainsi. Quand don Carlos évoque le «ressenti » comme la base du "savoir ", il ne l'oppose pas à la pensée. Selon lui, les émotions, tout comme les pensées, pénètrent le corps humain via les odeurs et les vents et se consolident dans la chuyma. Ces émotions passent par l'expérience et de fait, donnent lieu à une connaissance personnelle expérientielle, c'est-à-dire à une connaissance "ukanaw », la connaissance des choses telles qu'elles sont. Ainsi, lorsque j'ai demandé à don Carlos et à Antonio, le jeune intellectuel et activiste aymara mentionné plus haut, s'ils pensaient avec leur chuyma ou leur piqi, tous deux ont hésité puis m'ont donné des réponses forcées, dénuées de substance. À la fin de sa réponse, don Carlos est apparu fatigué de mes interrogations et m’a demandé : 
«Pourquoi des questions aussi stupides? Tu devrais t'y connaître bien mieux après toutes ces années! ». Je crois que c'est une situation que la plupart des anthropologues ont vécue : nos questions reposent sur des prémisses qui ne sont pas celles de notre interlocuteur. Dans ce cas précis, on voit bien que ma question reposait sur une fausse prémisse, précisément sur le fait que la piqi et la chuyma sont catégoriquement distinctes et qu'elles opèrent en des sphères différentes. De façon plus fondamentale, cependant, ma question se base sur une autre fausse prémisse qui voudrait que penser et savoir soient des opérations qui se jouent au sein d'un sujet autonome. La question toute entière se basait ainsi sur un dualisme ontologique initial entre deux mondes séparés : le monde intentionnel des sujets humains (ceux qui connaissent) et le monde réifié des choses matérielles (ce qui est connu). Don Carlos et Antonio soutiennent cependant qu'ils ne vivent que dans un seul monde, un monde en constant changement caractérisé par des contradictions politiques et des dynamiques sociales et culturelles auxquelles ils doivent s'adapter et qu'ils doivent parvenir à gérer lors de leurs déplacements entre contextes ruraux et urbains mais qui reste cependant un seul et même monde. Ils se disent aussi intimement connectés à d'autres sujets sages de ce monde.

14 Quand don Carlos et Antonio parlent en espagnol, ils évoquent souvent le fait « d'être connecté » (" estar conectado ») aux ajayus uywiris, achachilas et awichas, c'est-à-dire aux êtres ancestraux, sages et créateurs de vie du cosmos et du paysage andin. Au premier abord, j'ai cru que "connexion" représentait une sorte de relation qui liait deux entités plus ou moins distinctes. Cependant, quand j'ai réalisé qu'ils utilisaient les termes « mayisthapita " ou « mayachata » pour parler de la même chose en Aymara, ma compréhension du terme "connexion » a commencé à changer. "Mayisthapita » et " mayachata » évoquent tous deux le fait de "ne faire qu'un ». Être connecté aux ajayus uywiris sous-entend alors ne faire qu'un avec eux. En participant au même changement et rythme de vie rituels, les vents, les odeurs, le souffle, les ajayus uywiris et les humains ne font plus qu'un. On rencontre cette unité à ses moments les plus puissants dans certains lieux sacrés et sages (wak'as) et à des moments particuliers (rituels). Cette unité, de plus, nous dit quelque chose d'essentiel sur l'épistémologie aymara: non seulement nous pensons avec d'autres parties de notre corps que la tête mais en tant que sujets savants, nous ne sommes pas limités par notre peau. Non seulement nous pensons avec notre chuyma mais lorsque don Carlos réplique que «nous sommes dans la chuyma en ce moment " à ma question concernant la nature de la chuyma, il souligne le fait qu'en tant qu'êtres savants et doués de sensations, nous faisons partie de la chuyma de pacha. C'est-à-dire " qu'être dans la chuyma », c'est être pris par le cosmos et ne faire qu'un avec lui en même temps qu'avec d'autres êtres savants et doués de sensations, humains et non-humains, et de penser et savoir avec eux. Ainsi demander si l'on pense avec la tête ou avec les poumons n'est pas seulement une preuve d'ignorance mais aussi une question sans intérêt.

15 Lorsqu'Antonio me dit qu'il pense aussi bien avec sa tête qu'avec ses poumons, mais aussi avec la pacha, le cosmos, et avec certains lieux wak'a présents dans le paysage, il remet en question ce que l'épistémologie hégémonique a établi comme "penser » et «savoir». Et il va foncièrement plus loin que toute dichotomie sujet-objet. En ne faisant qu'un et en expérimentant avec et au sein de ce que l'épistémologie conventionnelle et dominante qualifierait « d'objet de connaissance », la connaissance ne doit pas prendre de détour avec les mots ; elle est en effet d'autant plus fiable quand 
elle s'abstient. Comme avancé par Ingold (2000: 99), « les conditions de la vérité (...) ne reposent pas sur la correspondance entre une réalité externe et ses représentations idéales mais sur l'authenticité de l'expérience elle-même ». Ne faire qu'un avec le sujet de la connaissance et expérimenter le monde en son sein génère en effet des expériences authentiques. La correspondance entre l'intérieur (la pensée et le langage) et l'extérieur (le monde matériel) n'est viable que dans une théorie représentationaliste de la connaissance et dans une dichotomie ontologique entre l'esprit et la nature (une épistémologie cartésienne). Ce dont nous parlons ici est différent; il s'agit d'une compréhension participative de la pensée et de la connaissance. Si le logocentrisme représentationaliste prétend que le langage humain apporte idéalement un accès direct à la « réalité ", alors ceci en serait l'opposé.

16 Ce que don Carlos et Antonio prônent est une épistémologie de l'engagement (Ingold 2000 : 216) ou une épistémologie relationnelle (Bird-David 1999) avec un point de départ ontologique d'unité. Cela signifie que la pacha, le cosmos, entre directement dans la constitution de nos subjectivités, "pas seulement comme source d'enrichissement mais aussi comme source de connaissance » (Ingold $2000: 57$ ). Pour le chaman et activiste don Carlos, les dimensions enrichissantes et productrices de la connaissance du monde sont intimement entrelacées. Les vents et les odeurs nous font penser et ressentir des choses car ils pénètrent nos corps via notre chuyma et s'intègrent en nous; ils pensent via, avec et au sein de nous et nous font expérimenter et ressentir le monde ; dans un sens, ils nous éduquent en ne faisant qu'un avec nous. Il en va de même pour les plantes, l'eau, les minéraux et les animaux. Ils nous éduquent tout en nous nourrissant. Ils nous font vivre et penser car ils pénètrent nos corps puis deviennent des parts intégrantes de nos êtres, que ce soit pour un moment ou de façon plus permanente. De la même manière, nous pensons et expérimentons via, avec et depuis la pacha et particulièrement via, avec et depuis les wak'as, les lieux sacrés et sages du paysage aymara. Connaître le monde, c'est le ressentir et ne faire qu'un avec lui. Ceci n'est bien sûr compréhensible que dans un cosmos lui-même savant, réceptif, doué de sentiments et dans lequel les relations sociales entre les êtres sages peuvent « surpasser les limites de l'humanité en tant qu'espèce » (Ingold 2000 : 107).

Une discussion autour de notions telles que celles-ci pourrait facilement devenir abstraite et éloignée de son contexte d'origine. Permettez-moi donc d'ancrer une nouvelle fois cette discussion dans un « monde de vie » aymara spécifique.

Wara est une femme d'origine aymara néée à La Paz sous la dictature Banzer dans les années 70. Ses grands-parents ont migré de la campagne vers les zones périphériques de La Paz dans les années 50, où ils ont obtenu des emplois peu rémunérateurs. Ses parents, forts de leur expérience de la discrimination et du racisme dus à leurs origines, ont pensé qu'il était plus sage de ne pas lui enseigner l'aymara. Ainsi, comme de nombreux hommes et femmes de l'environnement urbain et de son âge, Wara ne parle pas plus de quelques mots de la langue maternelle de ses grands-parents. Son sens de "l'identité aymara» est loin de s'exprimer clairement et il a donné lieu à des questionnements existentiels. Lorsqu'elle étudiait à l'université, les questionnements de Wara s'inscrivaient dans le cadre d'une bataille sociale et politique au sein du mouvement étudiant indianiste-katariste. Elle avait l'impression de posséder un « soi » refoulé et de s'être installée dans un monde refoulé. Plus tard, elle a identifié le colonialisme comme la source de son mal être. Pour Wara, le colonialisme se présente non comme un "autre» imposé mais plutôt comme un sentiment de perte, 
d'incomplétude et de mutilation physique. Démontrant comment les métaphores du colonialisme en tant que mutilation corporelle collective peuvent être vécues dans un corps individuel (voir ex. Burman 2009 : 121-2), elle analyse : «J'ai toujours ressenti un vide ou la sensation qu'une partie de mon corps me manquait, comme un pied ou un bras quelque part."

19 Se rapprocher des chamans aymara et s'essayer aux pratiques rituelles dans des lieux wak'a sacrés a été un moyen pour Wara de régler ce problème. Lorsqu'elle prépare une offrande aux êtres ancestraux et sages qui peuplent ces lieux, elle dirige ses pensées et effectue des postures et mouvements singuliers. À chacune des offrandes que Wara prépare, ses aptitudes rituelles s'améliorent et elle ressent que son propre sarawi, c'està-dire sa manière de faire les choses, finit par ressembler à l'idée qu'elle se fait du sarawi de ses ancêtres. Elle commence à se rapprocher, grâce à l'expérience, de l'ordre aymara «correct» au sein du monde et en elle. La complétude de l'offrande devient coextensive avec sa propre complétude. Wara dit: «Lors des rituels et dans les lieux sacrés, je me sens complète, je ressens la présence des ancêtres parmi nous, dans le cercle, et je sens que plus rien ne me manque désormais ».

Même l'étude ethnographique la plus approfondie et la mieux écrite ne suffirait pas à l'accomplissement de cette complétude. La pratique corporelle rituelle et l'expérience sont requises. La pratique rituelle engage et le «sarawi » et le « amtawi», faire et savoir, agir et se souvenir. Lorsqu'elle entreprend une pratique rituelle dans des lieux wak'a, Wara sent que son corps se meut en harmonie avec l'ordre du monde et qu'elle s'imprègne d'un système de connaissance " correct ». En se souvenant et en agissant en harmonie avec cette connaissance, elle est «re-membrée ». Pour Wara, il s'agit d'une expérience transformatrice que de ne faire qu'un avec la pacha, le cosmos, et avec ses propres origines. Lorsque les offrandes sont disposées puis brûlées, Wara sent que « les ancêtres la reconnaissent ». Cette reconnaissance est mutuelle. En quittant la ville, en escaladant les montagnes, en préparant les offrandes et en les disposant, Wara reconnaît la présence des achachilas et des awichas dans le paysage, que ce soit dans les pierres, les rochers, les montagnes, lacs ou animaux, et elle réaffirme leur statut d'êtres ancestraux. En acceptant ses offrandes, les achachillas et awichas reconnaissent Wara comme une personne et de façon plus importante, comme leur petite fille - une Aymara. Ainsi, détenir une vraie connaissance du monde et des êtres ancestraux qui le peuplent, c'est en avoir fait l'expérience, avoir partagé des choses et n'avoir fait qu'un avec eux et ainsi avoir pensé avec eux. Et détenir une vraie connaissance de la nature de sa propre existence, c'est avoir fait l'expérience de cette unité, ou, comme don Carlos et Antonio la nomme, mayisthapita, mayachata. Dans ce cas, la pratique rituelle dessine les conditions d'existence de la connaissance ; révéler la connaissance dans sa présence au monde et rendre les participants attentifs au monde. Ce que nous savons est inséparable de la façon dont nous le savons et l'on ne peut distinguer le « sachant » du «su ». La connaissance ne provient donc pas $d u$ monde mais intervient et s'épanouit parmi ses différents éléments de sagesse.

21 Il n'y a donc rien d'ésotérique dans le fait de "penser avec des lieux ", il s'agit plutôt d'une façon de produire de la connaissance au-delà des dichotomies cartésiennes telles que la nature-culture et l'objet-sujet et au-delà de l'hégémonie des épistémologies logocentriques et librocentriques. L'expérience est évidemment centrale dans l'épistémologie aymara. Cependant, comme nous allons le voir maintenant, un certain type de mots joue aussi un rôle crucial dans la production de la connaissance aymara ${ }^{3}$. 


\section{Mots médicinaux} n'importe quel vrai chaman. Ce sont des mots qui proviennent des poumons et non de la tête : ce sont des mots qui détiennent le pouvoir de guérir. Afin de comprendre ce que veut dire guérir dans le contexte aymara, il nous faut nous pencher brièvement sur les notions de maladie chez les Aymara (pour une étude plus poussée, voir Burman 2010). Le monde aymara est imbibé d'ajayus, généralement traduit par «esprits». L'ajayu est un pré-requis pour la circulation de la vie, une obligation pour toutes les variétés d'existences. Là où il y a de la vie, il y a l'ajayu. Cependant, l'ajayu aymara n'est en rien le « fantôme dans la machine » cartésien ni une " âme » dans le sens d'un cœur spirituel personnel permanent et stable. Les ajayus nous entourent. Ils imbibent le paysage et les lieux, l'être et l'existence, le corps et l'esprit, ils nous font vivre, ressentir et penser. Les êtres qualifiés de " déités andines » en ethnographie conventionnelle ne sont ni plus ni moins que des ajayus différents, supervisant et autorisant le cours de la vie chez toutes les formes d'existences.

Cependant, il existe aussi d'autres ajayus. Il s'agit d'ajayus dangereux qui font dévier les gens du cours de leur vie et les engage dans d'autres cours où la réciprocité n'existe pas. Il existe des ajayus «étranges » qui causent le désordre, la maladie ou même la mort. La plupart des maladies du monde aymara s'expliquent via un système de connaissance thérapeutique dans lequel ces ajayus «étranges » jouent un rôle crucial. Les personnes en bonne santé, sympathiques et intégrées socialement, le sont grâce à l'unité qu'elles forment avec les ajayus uywiris, c'est-à-dire les êtres ancestraux, bienveillants et protecteurs. De même, les personnes malades, agressives et asociales, le sont à cause de l'unité qu'elles forment avec les ajayus étranges. Cependant, ces derniers ne sont pas considérés comme des esprits totalement malveillants. Ils ne sont pas fiables et puisqu'ils ont le pouvoir de provoquer le malheur dans le monde, ils sont souvent assimilés aux oppresseurs blancs coloniaux, les $q^{\prime} \operatorname{aras}^{4}$; ils exploitent les autres, sont abusifs et «étranges ». De plus, ils pénètrent les corps des individus, leur esprit et poumons et provoquent la maladie ainsi que des opinions et des compréhensions biaisées du monde. Don Carlos l'explique ainsi : «Des esprits étranges les pénètrent, c'est pourquoi ils parlent mal. Sánchez de Lozada ${ }^{5}$ et [George W.] Bush en sont des exemples; cette personne est malade, cela explique pourquoi il tue des gens ». Cette citation révèle un fait intéressant que j'explore autre part (Burman 2009 et 2010) : les chamans aymara parlent souvent de colonialisme en termes de maladie (un pouvoir «étrange » provoquant le désordre et une opinion biaisée du monde). De même, ils parlent de décolonisation en termes de guérison.

Il existe de nombreuses pratiques différentes pour gérer les maladies provoquées par les esprits "étranges» mais l'utilisation des qulla arus, les mots médicinaux, est centrale chez beaucoup d'entre elles. Les qulla arus sont utilisés pour guider les gens vers les champs relationnels, descréateurs de vie du cosmos et pour les désengager des champs relationnels des esprits «étranges ». Ils permettent également de libérer la connaissance. Cependant les qulla arus servent non pas à transmettre de la connaissance aux gens mais à guider leur attention dans un monde de sujets sociaux savants, humains et non-humains, et à les amener à faire par eux-mêmes l'expérience des choses telles qu'elles sont, c'est-à-dire à créer de la "connaissance ukanaw", 
comme Antonio la nomme, ou à «sentir le monde » comme le dit don Carlos. Quand don Carlos parle qulla arus aux gens, rien n'est véritablement transmis. Cela se rapporte plus au fait de raconter une histoire dans laquelle l'attention de celui qui écoute est guidée, ce qui est une façon de diriger l'attention des gens sur certains chemins ( $\mathrm{cf}$ Ingold $2000: 190$ ). Ainsi les qulla arus sont des «instruments de perception» (Ingold $2000: 146)$; ils éduquent notre attention et nous font voir le monde plutôt que de simplement le regarder. En tant que tels, ils sont relativement différents du texte écrit conventionnel. Cependant, comme l'étude suivante le montre, don Carlos est fasciné par les mots écrits depuis sa plus tendre enfance.

Au début des années 60 dans la province rurale aymara de Omasuyos, Carlos se trouvait sur le long chemin du retour après toute une journée à l'école. Bien qu'il ait toujours des problèmes à comprendre son professeur monolingue espagnol, les quelques semestres passés à l'école lui avaient donné quelques bases d'écriture et de lecture. Et il était désireux de les utiliser. Sur tout le chemin du retour, il écrivit des noms d'animaux à la craie, sur des pierres sur le bas-côté de la route. Il écrivit «cóndor ", « zorro », « llama » juste parce qu'il aimait voir les mots sous la forme écrite. Une fois à la maison et au grand désespoir de sa mère, il écrivit les noms des animaux, plantes et personnes sur les murs d'adobe de la maison. Sa mère le réprimanda sévèrement.

Quarante ans plus tard, les velléités d'écriture de don Carlos prirent forme dans un livre merveilleux (Yujra 2005) intitulé Laq'a achachilanakan jach'a tayka amuyt'äwinakapa (les pensées maternelles de nos ancêtres). Il s'agit d'un livre bilingue d'abord écrit en Aymara par don Carlos puis traduit en espagnol par l'un de ses amis. Son livre attire notre attention sur la pacha tout entière, le cosmos aymara. Cependant, selon don Carlos, les livres ne contiennent aucun savoir. Et il n'entend pas cela dans le sens de Platon, par exemple, qui considérait le mot écrit comme une façade visible de la réalité sonore intérieure des mots prononcés (Ingold 2000 :247). Pour don Carlos, la distinction essentielle ne se fait pas entre la connaissance transmise par les mots prononcés et la connaissance transmise par les mots écrits mais entre la connaissance transmise par les mots et la connaissance transmise dans le monde. Les mots écrits par don Carlos ne représentent pas des choses, un monde ou des idées. Ses mots écrits sont des qulla arus qui attirent notre attention sur certaines particularités du paysage, comme les lieux wak'as sacrés et sages, et sur certaines pratiques, mouvements et événements et la façon dont ceux-ci sont reliés entre eux et à nous. Il s'agit d'une connaissance relationnelle, souvent dénuée de proposition; une connaissance non pas $d u$ monde mais en son sein, un type expérientiel de connaissance qui prend racine dans le fait de sentir le monde en son sein.

Une fois, j'ai demandé à don Carlos ce qui adviendrait de toute cette connaissance le jour où il mourrait. Il m'a paru décontenancé, $\mathrm{j}$ 'ai donc reformulé ma question : « Etesvous inquiet de ne pas avoir de disciple précis à qui transmettre votre connaissance?» Cette fois-ci, il a souri et a fait non de la tête puis a dit:

«Vous ne comprenez toujours pas, n'est-ce pas? Je ne peux la transmettre à qui que ce soit. Les gens doivent la ressentir par eux-mêmes. Je peux juste leur indiquer les lieux où ils devraient aller... ensuite ils s'y rendront, ressentiront et penseront les choses. Si c'est un bon endroit, de bonnes pensées leur viendront. »

Ainsi, don Carlos n'écrit pas et ne parle pas qulla arrus dans le but de transmettre une quelconque connaissance. Ses mots sont prononcés et écrits afin que les gens fassent eux-mêmes l'expérience de la source de sa connaissance et ne fassent plus qu'un avec cette même source. Ainsi les qulla arus ne représentent pas le monde; ils nous orientent 
dans notre expérience sensitive et procèdent aussi bien sous forme de texte écrit que de mots prononcés.

Ceci dit, dans la société bolivienne contemporaine, il n'y a bien évidemment aucune ligne de division absolue entre les théories hégémoniques de la connaissance et les épistémologies indigènes, entre la connaissance propositionnelle et nonpropositionnelle, entre la connaissance $d u$ monde et la connaissance au sein $d u$ monde ou entre les façons représentationnelles et relationnelles de savoir. On ne peut trouver de dichotomies si précisément définies après presque 500 ans de mélange asymétrique et colonial d'épistémologies et de systèmes de connaissance provenant de différentes traditions. De plus, les Aymara ont développé une capacité surprenante à gérer une logique et des pratiques apparemment contradictoires de façon simultanée. Cependant, ceci ne signifie pas que la culture aymara contemporaine est essentiellement « inclusive » et "ouverte " à n'importe quelle influence externe, comme l'avance le théologien et philosophe Josef Estermann (2006) lorsqu'il parle de la spiritualité aymara comme d'une réception fluide d'éléments chrétiens en une cosmologie aymara réceptive. Avancer de telles choses serait ainsi sous-estimer l'importance des relations de pouvoir coloniales présentes et passées. Reconnaître que les façons de savoir hégémoniques et indigènes s'entrecroisent dans les Andes depuis presque 500 ans ne devrait pas nous mener à ignorer le fait qu'au sein du contexte du système-monde colonial et de l'État moderne, les façons de savoir relationnelles et les traditions de pensée indigènes de ses institutions éducatives sont systématiquement infériorisées. Cependant, ces façons de savoir subalternes sont toujours là. Et dans les Andes boliviennes, elles font sentir leur présence à l'université.

\section{Des livres auxquels penser}

Peu après qu'Evo Morales et le Movimiento al Socialismo soient arrivés au pouvoir en 2006, la « décolonisation » fut proclamée comme l'une des visions politiques générales du nouveau gouvernement. L'éducation supérieure fut alors identifiée comme un point clef pour la mise en place, la génération et l'articulation de la politique de décolonisation. Rappelons que les revendications indigènes concernant l'éducation et les initiatives en Amérique du Sud se trouvaient de plus en plus transférées du champ de l'éducation basique à celui de l'éducation supérieure. Il faut comparer les revendications des années 1970 et d'après pour une éducation interculturelle et bilingue, revendications majoritairement abordées et neutralisées par le multiculturalisme néo-libéral, avec les revendications actuelles concernant la décolonisation de la connaissance et de l'université (López et. al. 2009 : 246). En Bolivie, l'une des plus grandes mobilisations visant à créer quelque chose de « correct » dans le domaine de l'éducation supérieure fut générée à la fin des années 90 par les demandes de la population, indigène ou non, pour une université dans la ville d'El Alto. Cela aboutit à la création de l'Universidad Pública de El Alto (UPEA). Un activiste aymara qui prit part à ces mobilisations en détaille le fonctionnement :

« Nous avons créé tout cela nous-mêmes. El Alto s'est élevé. Et je me souviens ces premiers mois à suivre les cours assis sur des blocs d'adobe alors que nos frères faisaient office de conférenciers sans aucune rétribution financière... »

Aujourd'hui, l'UPEA est une université très conventionnelle sur plusieurs aspects mais assez marginale sur d'autres. Elle l'est dans le sens où elle offre à ses étudiants la même 
gamme de critères académiques et de programmes que la plupart des universités en Bolivie ou ailleurs. Aucune pensée rhétorique visant à "retrouver les identités des nations indigènes ${ }^{6} »$ ne peut occulter cela. Pas plus que l'insertion d'une série de courts enseignements obligatoires sur "L'histoire des Nations indigènes » dans tous les diplômes. Cependant, UPEA est marginale dans le sens où une écrasante majorité de ses étudiants est d'origine aymara. Elle est marginale dans le sens ou les idéologies indianistes-kataristes imbibent ses organisations et activités estudiantines. Elle est aussi marginale dans son fonctionnement structurel interne : la voix et le vote de n'importe quel étudiant a le même poids que ceux de n'importe quel professeur. De plus, les organisations sociales d'El Alto ont eu leur mot à dire depuis la création d'UPEA en 2000. En résumé, les étudiants indianistes-kataristes de l'UPEA soulignent généralement le besoin d'une profonde décolonisation de leur université.

32 Avant que l'UPEA ne soit créée, il existait d'autres initiatives aymara luttant pour une éducation supérieure «correcte». El Instituto Tecnológico y de Investigación Andino (Inti Andino) situé dans la province de Gualberto Villarroel était l'une d'entre elles. J'ai travaillé comme enseignant pendant 2 ans, il y a dix ans de cela, dans cette ONG au programme explicitement indianiste-katariste. Je me souviens de tout le travail dédié à l'intégration au programme de sujets tels que la cosmologie andine, la philosophie andine, l'écologie andine, les droits indigènes et communautaires et l'agronomie andine traditionnelle.

Une autre université, l'Universidad Indígena Tawantinsuyu (UTA) s'inscrit elle aussi dans le mouvement indianiste-katariste fondé dans le petit village de Laja à la fin des années 90. Alors que l'UPEA, comme décrit plus haut, est conventionnelle sur certains aspects et marginale sur d'autres, UTA est marginale sur la plupart de ses aspects. L'UTA fut le lieu qui radicalisa Antonio, le jeune activiste et intellectuel aymara mentionné plus haut. À l'école primaire, Antonio ne remettait pas en question les doctrines de ses enseignants mais acceptait au contraire leurs opinions du monde comme correctes. Il explique comment il a absorbé l'histoire bolivienne officielle, comment il prêtait serment au drapeau et comment, lors de son service militaire, on lui apprit à défendre la patria de sa vie. À son retour dans sa communauté rurale natale après son service militaire, sa mère lui conseilla de faire des études. Non loin de sa communauté se trouvait l'UTA, gérée par Germán Chukiwanka, connu pour avoir été couronné Inca pendant l'apogée de la "Campagne des 500 ans de la Résistance » en 1992. Antonio décida alors de s'inscrire au diplôme de droits indigènes. Sa motivation ne venait pas de quelque conviction idéologique mais simplement de considérations pratiques et de la conviction que les études universitaires lui ouvriraient de nouveaux horizons et opportunités. Il ne savait encore rien de l'expérience transformative que ses études universitaires allaient représenter pour lui. Les professeurs tenaient des discours saisissants et encourageaient les étudiants à fouiller en eux-mêmes, souvent dans leur passé, pour trouver des solutions au lieu d'accepter simplement les vérités coloniales établies. L'environnement estudiantin était nourri par des débats constants, passionnés, et une pensée radicalisée. Antonio explique :

"C'était comme si quelqu'un m'avait versé un seau d'eau glacée sur la tête. J’ai alors compris que j'avais vécu jusque-là les yeux clos. Là-bas, à l'UTA, j'ai compris cela et j'ai pu voir le monde tel qu'il est, c'est-à-dire que la vraie connaissance réside dans la connaissance de nos ancêtres. »

34 À l'UTA, actuellement située dans la ville d'El Alto et ouverte seulement le week-end, les étudiants ont la possibilité d'étudier la théologie et la philosophie andines, les droits 
indigènes, le tourisme, l'histoire et la linguistique aymara. Bien que certaines de ces matières puissent paraittre conventionnelles, elles ont toutes pour but de prendre « les traditions de pensée indigènes " comme point de départ fondamental et non comme supplément culturaliste dans un parcours universitaire conventionnel. De plus, non seulement les universitaires aymara attestant d'un parcours académique plus ou moins conventionnel enseignent à l'UTA mais les activistes et chamans sont également invités à partager leurs expériences dans la salle de conférence. L'UTA est donc une université relativement marginale.

Le petit village aymara de Warisata est l'une des références fondamentales pour n'importe quel type d'initiative critique indigène liée à l'éducation. Le 2 août 1931, une école unique y fut créée; l'Escuela-Ayllu de Warisata. L'école adopta comme point de départ les réalités indigènes sociopolitiques et économiques de l'époque et se vit rapidement immergée dans un questionnement strict du caractère colonial de la société bolivienne du $\mathrm{xx}^{\mathrm{e}}$ siècle puis fit le projet de libérer « el indio ». Cependant, après quelques années seulement, l'école fut fermée par les autorités de l'État et ses bâtiments furent utilisés pour l'entraînement conventionnel des enseignants sous le contrôle de l'état. L'école devient un instrument utilisé pour intégrer les peuples indigènes dans la société bolivienne (voir Luykx 1999).

Ceci dit, les temps changent et lors du $77^{\mathrm{e}}$ anniversaire de la création de la Escuela-Ayllu, une université financée par l'état fut créé à Warisata par le premier président indigène de Bolivie, Evo Morales Ayma. L'Universidad Indígena Boliviana aymara «Tupak Katari » à Warisata propose les matières suivantes: agronomie, sciences vétérinaires et zoologiques, industrie textile et industrie alimentaire. On dit que l'université indigène est un pas vers la décolonisation de l'éducation supérieure. Ainsi, le cercle est apparemment fermé et l'éducation semble être devenu un outil, non pas pour l'assimilation coloniale mais pour l'émancipation indigène, à la façon de l'EscuelaAyllu dans les années 30.

Cependant, l'étude suivante révèle quelques tensions et ambiguïtés qui caractérisent le projet de décolonisation de l'éducation supérieure. En septembre 2009, j'ai été invité par deux de mes amis - des intellectuels radicaux non-indigènes, de classe moyenne et issus de l'administration Morales - à les accompagner à un séminaire sur la "décolonisation et l'éducation " à l'Institut de l'Education à Warisata, à deux pas de l'université indigène récemment implantée. Nous nous y sommes donc rendus et un groupe d'étudiants aymara enthousiastes nous ont accueillis. Le séminaire s'est bien déroulé; mes deux amis, représentants de l'Etat, ont échangé sur les travaux des penseurs critiques Enrique Dussel, Franz Hinkelammert, Paulo Freire et d'autres, et les étudiants aymara les ont écoutés attentivement. Tout en écoutant le séminaire, j'ai regardé vers l'ouest par la fenêtre et j'ai été frappé par la présence à l'horizon, à quelques kilomètres seulement, de Pachjiri, une montagne ancestrale et exceptionnellement puissante. Combien de fois n'avais-je pas grimpé cette montagne avec les chamans ? Combien de fois n'avais-je pas rencontré le lever de soleil là-haut après des nuits entières de rituels sur la montagne? Il s'agit en effet d'un lieu de sagesse où et avec lequel chacun, selon les termes de don Carlos, peut « avoir de bonnes pensées" et obtenir une "bonne connaissance». Elle se tenait là, visible depuis la fenêtre de la salle de conférence où l'on débattait de problèmes comme la décolonisation, la connaissance et l'éducation. Beaucoup de livres importants et 
d'écrivains connus furent mentionnés mais jamais dans la discussion n'apparut le nom de Pachjiri ou d'aucun autre wak'a.

Sur le chemin du retour après notre visite à Warisata, je montrai du doigt l'arête de la montagne où Pachjiri se tient et demandai à mes amis s'ils connaissaient cet endroit. Ils firent non de la tête; je me mis alors, là dans le bus, à formuler toute une série de questions qui, in fine, me conduisirent à écrire cet essai. Si la décolonisation de la connaissance se trouve être essentiellement une question de théorisation intellectuelle " critique », s'il ne s'agit que de livres, de conférences et de mots, si les épistémologies indigènes sont méprisées ou simplement ignorées dans la pratique précise qui tente expressément de se débarrasser du déséquilibre épistémologique de l'ordre mondial colonial actuel, n'y a-t-il pas un risque que le "projet de décolonisation » finisse par soutenir les asymétries épistémologiques au lieu de les défier et de les ébranler? N’y at-il pas un risque que la décolonisation de la connaissance soit convertie en un projet d'universitaires et intellectuels mondains, un projet de l'université, un projet logocentrique et librocentrique? En d'autres termes, où cela situe-t-il don Carlos, Antonio et Wara, les trois personnes aymara que j'ai présentées ici, et leur connaissance et expériences? Où demeurent les montagnes, les lacs, les arbres, le condor, le renard, les rochers et pierres, et leur connaissance et expérience ?

Les épistémologies relationnelles indigènes ont une longue histoire au sein des Andes boliviennes. Cependant, les notions logocentriques et librocentriques de connaissance justifient également d'une histoire et d'une place centrale quand il s'agit de production de connaissances politiquement et académiquement reconnues, même dans la production qui d'une façon ou d'une autre pose la question de l'hégémonie coloniale dans la société bolivienne. Faustro Reinaga, l'idéologiste indianiste du 20 ème siècle, l'homme qui, dès les années 1960, a imaginé une Universidad India et a exprimé le besoin d'une Revolución India (voir Reinaga 2001 [1970]), en est un exemple. L'apport idéologique de Reinaga dans le mouvement indianiste-katariste contemporain est vaste. Cependant, dans ses travaux, Reinaga est plus absorbé par une critique philosophique des penseurs et textes européens par telle ou telle tradition de pensée indigène. Il y a quelques passages extraordinaires dans ses écrits où il parvient à réexaminer la pensée européenne à partir de conceptions indigènes, mais ils sont assez rares. Généralement, il critique la philosophie européenne via une pratique philosophique peu éloignée de celle qu'il dénonce. De plus, Reinaga a souvent dit que 14000 livres avaient été dérobés de sa bibliothèque personnelle en 1972 par la dictature militaire d'Hugo Banzer. Les livres étaient en effet centraux dans son projet indianiste.

Les livres sont tout aussi importants pour le vice-président bolivien Alvaro García Linera dont la bibliothèque personnelle égale presque celle de Reinaga en termes de quantité. On dit qu'elle compte à peu près 10000 livres et, du moins avant de prêter serment comme vice-président, on disait qu'il lisait au moins six heures par jour ; Karl Marx étant, bien entendu, l'une de ses lectures favorites. Juan José Bautista est une autre figure de l'intelligentsia critique bolivienne qui se bat pour le besoin de «penser par soi-même " mais proclame en même temps que " quiconque veut faire une critique précise du cadre catégoriel de la pensée moderne-postmoderne ne peut le faire sans Hinkelammert, tout comme il ne peut le faire sans Marx, s'il ne veut pas paraître naïf " (2007:70, ma traduction). Ceci est une déclaration problématique selon moi. Cela l'est car le "cadre catégoriel de la modernité" est non seulement critiqué mais actuellement transcendé par les chamans, activistes et penseurs aymara. La critique 
exposée, par exemple, par don Carlos, prend racine dans la connaissance expérientielle. Il n'a jamais entendu parler de Hinkelammert et ne connaît pas les écrits de Marx. Cela dit, il n'y a rien de naif dans sa critique de la modernité. Mais alors sa critique n'est pas une «critique précise du cadre catégoriel» de la modernité, mais une critique qui désobéit et dénie l'autorité d'un tel cadre.

Dans beaucoup d'études universitaires s'intéressant à la connaissance, aux peuples indigènes et à la décolonisation, on observe une tendance à se référer à Marx et à Hinkelammert afin de comprendre les dynamiques coloniales et décoloniales en place en Bolivie et ailleurs. Mais penser que l'on puisse comprendre ces dynamiques sans prendre au sérieux la production de la connaissance et les moyens spécifiques de connaître certains lieux s'apparenterait selon moi à de la naïveté. Cependant, l'on a l'impression que les livres, les penseurs européens et les structures épistémologiques hégémoniques modernes empêchent les façons indigènes d'appréhender le savoir d'être prises au sérieux.

Le ministre des affaires étrangères bolivien et activiste aymara Davíd Choquehuanca, bien que souvent moqué, est un souffle d'air frais dans ce contexte ; il revendique le fait de ne jamais lire de livres mais préfère à la place lire, comme il le dit, « les rides sur les visages de nos anciens». Il considère les anciens comme des «bibliothèques ambulantes » et parle des restes lithiques des sociétés andines pré-coloniales comme de «livres de pierre ${ }^{7}$ ».

De façon intéressante, ces deux tendances, celles respectivement incarnées très explicitement par Bautista et Choquehuanca, sont présentes dans les universités indigènes en Bolivie. Et les deux disent traiter de la décolonisation de la connaissance. Dans les universités indigènes, on peut même expérimenter la façon dont les suppositions ontologiques et épistémologiques diffèrent d'une salle de classe à l'autre et même d'un moment à l'autre dans la même classe. Que signifierait alors décoloniser la connaissance et l'université et qu'est-ce qu'une université indigène?

Quand je parle de ces sujets avec Antonio, il se base sur deux points de vue apparemment contradictoires. D'un côté, il souligne fortement l'importance de " penser par soi-même » dans la langue aymara, dans les catégories aymara et selon les façons aymara, c'est-à-dire pas en tant que sujets individuels autonomes détachés du monde mais comme sujets relationnels pensant et produisant de la connaissance avec le monde et en son sein. De l'autre côté, il reconnaît l'importance de se sentir à l'aise avec les façons librocentriques conventionnelles de penser et de produire de la connaissance. La contradiction n'est bien entendu qu'une apparence. Il n'y a rien d'étrange ou de contradictoire dans le fait d'utiliser les mots et les pratiques académiques de la tradition coloniale hégémonique afin de contester la légitimité du pouvoir colonial. Comme je l'expose autre part (Burman 2010), les peuples indigènes ont développé des compétences pour redonner un sens à des concepts coloniaux " étranges » et pour ensuite les utiliser afin de contrer le pouvoir colonial. Le défi pour les universités, enseignants et étudiants indigènes est d'utiliser la pensée et le langage hégémoniques coloniaux mais sans adhérer complètement aux présuppositions épistémologiques et ontologiques de l'université coloniale conventionnelle, car il y a un risque que « le monde paraisse être ce que les catégories (...) européennes de pensée veulent te faire dire qu'il est » (Mignolo 2005 :36).

Il existe une notion omniprésente chez les étudiants et enseignants issus de différentes initiatives aymara pour l'éducation supérieure: le terme "indigène" présent dans 
" université indigène " ne soulève pas simplement une question de présence physique indigène, dans le sens où les enseignants et les étudiants sont d'origine indigène, car alors même UMSA à La Paz (pour ne pas mentionner UPEA à El Alto) remplirait les conditions pour devenir une université indigène puisqu'une majorité de ses étudiants pourraient sans doute être considérés comme indigènes. Il doit forcément y avoir quelque chose de plus à cela que la présence indigène, disent-ils. La production et la pratique intellectuelles dans une université indigène devraient être des outils contestatarios du système; elles devraient être insubordonnées, rebelles et désobéissantes. De plus, on avance le fait que l'université indigène devrait travailler avec, enseigner et produire une connaissance "correcte»; elle devrait créer de la connaissance en dehors des cadres hégémoniques de la rationalité moderne; elle devrait être un lieu d'apprentissage par le désapprentissage et le réapprentissage ; et tout ceci devrait vraiment se refléter dans son programme. En ce moment, on constate une présence physique indigène massive et beaucoup de désobéissance épistémologique parmi les étudiants comme parmi certains enseignants, mais il existe toujours, dans une large mesure, un programme "colonial». C'est le cas dans les universités conventionnelles, bien évidemment, mais également dans les universités indigènes jusqu'à un certain degré. L'UTA pourrait peut-être faire figure d'exception car la "pensée andine " y a une influence plus décisive sur cette génération précise de contenus universitaires. Même à l'université indigène de Warisata, une matière telle que Cosmovisión Andina, qui prend racine dans une tradition de pensée indigène, est encore incluse dans le programme général qui porte le sceau de l'université conventionnelle, même si c'est à un niveau inférieur qu'à l'UPEA ou l'UMSA par exemple. De plus, même si des "prácticas " sont menés par les étudiants, ce sont plus des applications de la connaissance reçue dans la salle de conférence que la façon fondamentale de générer de la connaissance et d'arriver à raisonner avec le monde et en son sein. La pratique académique générale se concentre toujours sur les livres, les mots et les conférences, c'est-à-dire le type de connaissance qu'Antonio appelle siwsawi, les opinions et spéculations entendues, dites et lues.

J'ai débattu plus haut du fait qu'il y a toujours un risque dans l'utilisation du langage et des théories académiques hégémoniques puisqu'elles peuvent nous empêcher de voir au-delà des présuppositions épistémologiques et ontologiques de la modernité coloniale. J'ai aussi débattu du fait que le programme universitaire est, jusqu'à un certain point, encore conventionnel même dans les universités indigènes. La solution serait alors de décoloniser le cursus universitaire en transformant le contenu pour que les traditions de connaissance et de pensée indigènes puissent être enseignées de façon plus complète dans les salles de conférence. Cependant, et j'identifie ici un second risque, si la connaissance indigène est intégrée à l'université, on peut se retrouver à coloniser la connaissance indigène au lieu de décoloniser l'université. Permettez-moi de vous exposer ce que j'entends par là :

Quand Elisabeth Croll et David Parkin (1992) prétendent que les peuples indigènes, leur environnement et leur existence existent inséparablement " au sein de l'un et l'autre ", ils soulignent quelque chose d'une importance fondamentale dans cette discussion. Institutionnaliser la connaissance aymara au sein des quatre murs coloniaux de l'université serait la décontextualiser et l'arracher au contexte précis et aux pratiques dans lesquelles et à partir desquelles elle est produite et se transforme au fil du temps. Cela voudrait alors dire séparer « la culture du lieu » (Ingold 2000 :225) ce qui altèrerait fondamentalement la nature de l'épistémologie aymara. De plus, n'importe quelle 
tentative de standardisation de la connaissance aymara reviendrait à inscrire celle-ci dans un cadre qui n'est pas le sien, à réifier et bloquer une cosmologie et ainsi altérer sa qualité dynamique. Il y a eu plusieurs tentatives de compiler des dossiers, pareils à des dictionnaires, de connaissance aymara "traditionnelle", telle que la signification des rêves (une source importante de connaissance). Mais de telles tentatives négligent le fait que la connaissance aymara n'est pas une série standardisée de données qui peut être transmise via les mots écrits et prononcés. La connaissance aymara est premièrement de type expérientelle. Connaître la signification des rêves, c'est avoir rêvé et ainsi avoir fait l'expérience de certaines occurrences de ce monde. Cependant, ce n'est pas comme si les mots de personnes sages n'étaient pas importants. Ils le sont très certainement mais ils représentent surtout des indications, conseils et incitations à faire l'expérience des choses par soi-même ; ils ne devraient pas être considérés comme de la connaissance ou même comme des outils de transmission de la connaissance. Où cela place-t-il l'université librocentrique ? Est-il possible de penser avec un livre de la même façon qu'avec un lieu, une montagne par exemple? Selon don Carlos et Antonio, penser avec un livre se résume à penser avec les suppositions et opinions des autres. On peut certainement utiliser ces suppositions dans la vie quotidienne mais si celles-ci sont d'ordre colonial, on risque de se retrouver avec des pensées coloniales. Les montagnes n'ont pas de pensées coloniales. Ainsi, il vaut mieux penser aux livres qu'avec eux.

\section{Conclusion}

Boaventura de Sousa Santos a avancé que «l'un des échecs de la théorie moderne a été de ne pas reconnaître que la raison qui critique ne peut être la même que la raison qui pense, interprète et légitime cela, ce qui est répréhensible » (2008:28, ma traduction). Je suis tout à fait d'accord avec lui. Quelques ajustements dans nos programmes académiques ou dans nos listes de références ne suffisent pas à engager des révoltes paradigmatiques, politiques, théoriques, épistémologiques et, finalement, cosmologiques. On ne ferait que reproduire les images coloniales et les "vérités " auxquelles les catégories hégémoniques réduisent le monde. Apprendre à penser avec d'autres catégories et en leur sein est un bon départ mais d'autres catégories ne feront pas tanguer nos piliers ontologiques. Afin que cela se produise, d'autres expériences sont nécessaires. En d'autres termes, il n'y a aucun moyen de nous faire un chemin hors de la colonialité par la raison intellectuelle, dans aucun sens académique conventionnel que ce soit. Il n'y a aucun moyen de nous sortir de la modernité en publiant des écrits. On ne se séparera pas de l'hégémonie épistémologique par la lecture.

Antonio dit qu'il existe une autre voie. Il raconte qu'il y a deux ans, lorsque sa mère et lui s'apprêtaient à réunir leurs vaches pour la nuit, celle-ci s'est arrêtée sur le versant de la colline et a ramassé une fleur pour en inhaler le parfum. Elle a dit «chuym qhanartayitu » : " ceci ouvre mon cœur à la lumière ». Quand Antonio se remémore cet épisode quelques années plus tard, il dit : « une odeur peut vous faire voir le monde tel qu'il est ; ceci est de la connaissance, c'est la connaissance de nos ancêtres ".

Ne voyez là aucun point de vue anti-intellectuel romantique. Ce que je fais, c'est de remettre en question la conception uni-dimensionnelle de la connaissance telle que reproduite par l'université. De plus, je questionne l'idée qu'un projet de décolonisation abstrait, logocentrique et librocentrique puisse être apte à transcender cette 
unidimensionnalité. Mon intention n'est pas de dénier l'importance des livres et des mots mais de les remettre à leur place et de d'examiner de façon critique les présuppositions épistémologiques de la pratique académique conventionnelle, considérées comme acquises.

51 Mon but n'est pas de critiquer les universités indigènes en Bolivie mais plutôt le contraire, je les soutiens au maximum. Face aux attaques constantes des universitaires conservateurs de droite, je défends évidemment la désobéissance épistémologique et la production critique de la connaissance qui prennent place dans ces universités et ailleurs. Ceci dit, nous ne faisons aucune faveur au soit-dit "processus de changement " ou à l'administration d'Evo Morales en applaudissant chacun de leurs mouvements sans émettre de critique. Les universités indigènes ne sont pas une sorte de paradis décoloniaux. Il existe, bien évidemment, une asymétrie épistémologique coloniale même dans ces universités. Ne pas admettre cela serait une preuve de naïveté. Cependant, ce serait faire preuve de cynisme que de ne pas reconnaître le potentiel des universités indigènes dans la mise en avant des racines coloniales des théories modernes de la connaissance, dans la capacité à désapprendre, à réapprendre et dans l'acquisition de compétences dans l'art de questionner les vérités coloniales gravées dans la roche.

\section{BIBLIOGRAPHIE}

Bird-David, N., “Animism Revisited : Personhood, Environment and Relational Epistemology." Current Anthropology (Supplement : Special Issue : Culture. A Second Chance ?), vol. 40, pp. 67-91, 1999.

Bautista, J.J., Hacia una Crítica Ética del Pensamiento Latinoamericano: Introducción al pensamiento crítico de Franz J. Hinkelammert. La Paz : Grupo Grito del Sujeto, 2007.

Burman, A., "Chachawarmi : Silence and Rival Voices on Decolonization and Gender Politics in Andean Bolivia." Journal of Latin American Studies, vol. 43, no. 1, pp. 65-91, 2011.

Burman, A., "The Strange and the Native : Ritual and Activism in the Aymara Quest for Decolonization." Journal of Latin American and Caribbean Anthropology, vol. 15, no. 2, pp. 457-475, 2010.

Burman, A., "Colonialism in Context : An Aymara Reassessment of 'Colonialism,' 'Coloniality' and the 'Postcolonial World'." Kult (Special Issue : "Epistemologies of Transformation : The Latin American Decolonial Option and its Ramifications”), vol. 6, pp. 117-129, 2009.

Croll, E. \& Parkin, D., Bush Base -Forest Farm : Culture, Environment and Development. London and New York : Routledge, 1992.

Dussel, E., 1492, El encubrimiento del Otro : Hacía el origen del "Mito de la modernidad." La Paz : Biblioteca Indígena, Vicepresidencia del Estado Plurinacional de Bolivia, 2008.

Estermann, J., Filosofía andina : Sabiduría indígena para un mundo nuevo. La Paz : ISEAT, 2006. 
Hardman, M., "Data-Source Marking in the Jaqi Languages." In W. Chafe \& J. Nichols (eds.), Evidentiality : The Linguistic Coding of Epistemology. Norwood, NJ : Ablex Publishing Corp, 1986, Pp. 113-136.

Ingold, T., The perception of the environment : Essays in livelihood, dwelling and skill. London and New York : Routledge, 2000.

Jensen, L., "Scandinavia-A Peripheral Centre.” Kult (Special Issue : "Epistemologies of Transformation : The Latin American Decolonial Option and its Ramifications"), vol. 6, 2009, pp. 161-179.

Lander, E. (ed.), La colonialidad del saber. Buenos Aires : CLACSO, 2000.

López, L.E., Moya, R. \& Hamel, R.E., "Pueblos indígenas y educación superior en América Latina y El Caribe.” In L.E. López (ed.), Interculturalidad, educación y ciudadanía : Perspectivas latinoamericanas, La Paz : FUNPROEIB Andes and Plural Editores, 2009, Pp. 221-289.

Luykx, A., The Citizen Factory : Schooling and Cultural Production in Bolivia. Albany : State University of New York Press, 1999.

Mignolo, W., The Idea of Latin America. Malden and Oxford : Blackwell Publishing, 2005.

Orta, A., "Syncretic subjects and Body Politics : Personhood, Doubleness and Aymara Catechists." American Ethnologist, vol. 26, no. 4, pp. 864-889, 1999.

Platt, T., "Writing, Shamanism and Identity, or Voices from Abya-Yala." History Workshop Journal, vol. 34, pp. 132-147, 1992.

Quintanilla, V.H., "La descolonización intra-intercultural de la filosofía en "Latinoamerica." In D. Mora (ed.), Interculturalidad crítica y descolonización : Fundamentos para el debate. La Paz : III-CAB, 2009, Pp. 127-147.

Reinaga, F., La revolución india. La Paz : Ediciones Fundación Amáutica Fausto Reinaga, (2001) [1970].

Rivera, S., Oprimidos pero no vencidos : Luchas del campesinado aymara y qhechwa 1900-1980. La Paz : Aruwiyiri, (2003) [1984].

Said, E., Orientalism. New York : Vintage Book, 1978.

Santos, B. de S., Conocer desde el Sur : Para una cultura política emancipatoria. La Paz : CLACSO, CIDESUMSA, Plural Editores, 2008.

Viveiros de Castro, E., Comments to Bird-David's “Animism Revisited : Personhood, Environment and Relational Epistemology." Current Anthropology (Supplement : Special Issue : Culture. A Second Chance ?), vol. 40, 1999, pp. 67-91.

Walsh, C., Schiwy F. \& Cástro, S. (eds.), Indisciplinar las ciencias sociales : Geopolíticas del conocimiento y colonialidad del poder. Perspectivas desde lo Andino. Quito : Universidad Andina Simón Bolívar y Abya-Yala, 2002.

Yujra, C., Laq'a Achachilanakan Jach'a Tayka Amuyt'äwinakapa : Los Grandes Pensamientos de Nuestros Antepasados. La Paz : CAUP, 2005. 


\section{NOTES}

1. J'utilise le terme "chaman " comme terme générique pour désigner une grande variété de spécialistes rituels et cérémoniels Aymara avec, par exemple, le yatiri (celui/celle qui sait), le qulliri (guérisseur), le ch'amakani (possesseur des ténèbres), le amawt'a (le sage).

2. L'indianisme et le katarisme sont des idéologies ethnopolitiques qui ont imprégné les dernières décennies des mobilisations et combats indigènes dans les Andes boliviennes. Afin de comprendre la montée du mouvement indianiste-katariste en Bolivie, il faut examiner (1) l'expérience post-révolutionnaire Aymara qui a poursuivi la marginalisation sociopolitique, la citoyenneté de seconde classe ainsi que la discrimination et (2) la mémoire collective de servitude coloniale et de rébellion indigène (cf. Rivera 2003).

3. De plus, Tristan Platt (1992: 138) et d'autres ont montré qu'on perçoit les mots écrits sous la forme de documents historiques comme détenteurs de pouvoir, ce qui pousse les autorités indigènes à les conserver précieusement comme fondements de la légitimité historique et des droits à la terre commune. Cependant, ces dynamiques s'éloignent de la visée de cet essai.

4. Q'ara est le terme Aymara qui désigne les Boliviens de descendance européenne. Sa signification littérale est "décharné", ce qui se réfère, selon les Aymara, à l'arrivée des Espagnols en Bolivie d'alors « sans rien, ni femme, ni objets personnels, ni terre », autrement dit « décharnés ». Les dominants sont socialement et culturellement « décharnés ».

5. Gonzalo Sánchez de Lozada, «Goni », fut président de la Bolivie à deux reprises. Son premier mandat s'étala de 1993 à 1997, et son second de janvier à octobre 2003, lorsqu'un grand soulèvement indigène l'obligea à démissionner et à fuir vers les Etats-Unis. Sánchez de Lozada fait actuellement l'objet d'une enquête pour avoir violé les droits humains et pour les actes génocidaires commis lors de ses dernières semaines au pouvoir.

6. http://www.elalto.galeon.com/upea.htm (consulté le 24 mai 2010).

7. Voir ex. http://www.wiphala.org/david.htm (consulté le 24 mai 2010).

\section{RÉSUMÉS}

Beaucoup d'hommes et de femmes aymara affirment que le savoir humain, transmis par la langue, n'est qu'un pur 'siwsawi', i.e. des paroles, des opinions, des points de vue, des jugements d'individus particuliers. Ainsi, il s'agit d'un savoir particulier ; c'est une connaissance concernant l'opinion de certaines personnes, rien de plus. Ceci est, de manière significative, différent du savoir non-linguistique, du savoir expérimental qui est vécu à travers et acquis dans, par, avec et au sein du monde. Ce type de savoir est 'ukamaw', la manière d'être. Les grandes lignes de ce raisonnement ont été établis par des linguistes intéressés par les dynamiques de 'l'évidentialité'/ evidentiality, i.e. les mécanismes par lesquels les locuteurs communiquent la fiabilité des savoirs qu'ils énoncent. Il a été montré que les orateurs aymara utilisent constamment des 'marqueurs de données linguistiques' pour indiquer s'ils parlent à partir de leur savoir personnel expérimental, du savoir obtenu par la langue ou d'un savoir non-personnel. Ici les deux premières catégories vont être discutées plus précisément.

Quand des personnes font une distinction entre le 'siwsawi', savoir acquis à travers le langage humain et l'ukamaw', savoir personnel acquis à travers l'interaction non-linguistique avec d'autres éléments du monde, porteuses de connaissances, elles utilisent la logique linguistique fondamentale Aymara. Dans la société Aymara, le non-respect des marqueurs linguistiques pour 
indiquer à partir de quel type de savoir on s'exprime est perçu avec suspicion. Quand des professeurs d'université ne le respectent pas, quand ils semblent affirmer avoir une connaissance personnelle sur tel sujet pour avoir lu un livre sur le thème, les étudiants Aymara tendent à conclure que des conférences et des livres sont suffisants si vous êtes intéressés par les opinions et les jugements des gens, mais que ce ne sont rien de plus que des siwsawi; des mots dits, écoutés et lus, pas des expériences vécues.

Cette façon de distinguer différents types de savoir, d'après leurs sources et leur fiabilité, a d'intéressantes implications dans l'actuel processus de décolonisation de l'Université Bolivienne et dans la récente création «d'universités indigènes » en tant que parties intégrantes des politiques étatiques de décolonisation lancées par l'administration d'Evo Morales.

Dans cette communication, je pose la question suivante : si les livres et les conférences sont une affaire d'opinions et de jugements, et que le savoir véritable ne peut être acquis que par l'expérience, non-linguistique, en inter-relation avec et dans le monde, le projet de décolonisation du savoir et des universités précisément par le biais de livres et de conférences, n'est-il pas un projet logocentrique et ethnocentrique de décolonisation, une entreprise prédestinée à reproduire les asymétries épistémologiques coloniales de la production du savoir? D'un côté, ce travail soulève les problèmes liés à la nature 'siwsawi' du savoir académique conventionnel (colonial) en rapport avec le processus critique de décolonisation. D’un autre côté, il explore l'ukamaw', savoir expérimental pour proposer une transformation épistémologique décoloniale de l'Université Bolivienne.

Fondamentalement, ce travail soulève des questions comme ce que veut dire " connaître ", être un sujet connaissant et appréhendable par la connaissance dans les Andes Boliviennes aujourd'hui, dans un contexte où les traditions subalternisées de la pensée sont un nouvel enjeu dans les récentes politiques d'éducation et où différentes visions et revendications de la vérité coexistent, fusionnent et s'entre-choquent.

Many Aymara men and women claim that human knowledge as transmitted through language is pure 'siwsawi', i.e. talk, opinions, views and judgments of particular individuals. As such it is knowledge of a particular kind; it is knowledge concerning the opinions of other humans, nothing else. It is thereby significantly different from the non-linguistic, experiential knowledge that is lived-through and gained in, from, with and within the world. This kind of knowledge is 'ukamaw', the way things are.

Lines of reasoning such as these have been addressed by linguists interested in the dynamics of 'evidentiality', i.e. the way languages provide an array of mechanisms for communicating how people regard the source and trustworthiness of their knowledge. It has been shown that Aymara speakers constantly use linguistic 'data-source marking' in order to indicate whether they are speaking from personal experiential knowledge, from knowledge acquired through language, or from non-personal knowledge. In this paper, the two first categories will be discussed in some detail. When people distinguish between the 'siwsawi' nature of the knowledge acquired through human language and the 'ukamaw' nature of the personal knowledge acquired through nonlinguistic interaction with other knowledgeable subjects in the world, they use a fundamental Aymara linguistic logic. In Aymara society, the failure to indicate from what kind of knowledge one speaks is looked upon with suspicion. When university lecturers fail to do so, e.g. when they seem to claim to have personal knowledge of a certain topic for having read a book about it, Aymara students tend to come to the conclusion that lectures and books are good enough if you are interested in people's opinions and judgments, but they are no more than siwsawi; words said, heard and read, not experience lived.

Thus, this way of distinguishing between different kinds of knowledge according to their source and supposed reliability has interesting implications for the current process of decolonization of the Bolivian University and the recent establishment of 'indigenous universities' as integral parts 
of the decolonizing state politics launched by the Evo Morales administration. In this paper I pose the question that if books and lectures are basically about the opinions and judgments of particular individuals, and proper knowledge is to be gained only in the experiential, nonlinguistic, inter-relational dealings with and in the world, wouldn't a project aimed at decolonizing knowledge and decolonizing the University precisely by way of books and lectures, i.e. a logocentric project of decolonization, be a venture fated to reproduce the colonial epistemological asymmetries of knowledge production? On the one hand, then, this paper scrutinizes the problems linked to the 'siwsawi' nature of conventional (colonial) academic knowledge in relation to a critical process of decolonization. On the other hand, it explores the 'ukamaw' nature of experiential knowledge and the prospects for this kind of knowledge to lay the fundaments for a decolonial epistemological transformation of the Bolivian University. Fundamentally, this paper addresses issues such as what it means to know, what knowledge is, and what it means to be a knowing and knowledgeable subject in the Bolivian Andes today, in a context where subalternized traditions of thought gain new urgency in new educational and political dynamics and where different visions and claims of truth coexist, coalesce and collide.

Muchos hombres y mujeres Aymara afirman que el saber humano, transmitido por la lengua, solo es un puro 'siwsawi', i.e. palabras, opiniones, puntos de vista, juicios de individuos particulares. Entonces, se trata de un saber particular; es un conocimiento que viene de la opinión de algunas personas, nada más. Esto es, de manera significativa, diferente del saber no-linguístico, del saber experimental que se vive a través y que se adquiere en, por, con y adentro del mundo. Este tipo de saber es 'ukamaw', la manera como son las cosas.

Fueron linguistas interesados por las dinámicas de la "evidencialidad" i.e. la voz de los lenguajes que vienen de una gama de mecanismos para transmitir como la gente considera la fuente y la fiabilidad de su saber, quienes establecieron las grandes líneas de este razonamiento. Ha sido demostrado que los oradores Aymara usan siempre "marcadores linguísticos" para indicar cuando hablan desde su saber personal experimental, del saber obtenido por la lengua o desde un saber no-personal. En esta presentación, las dos primeras categorías van a ser discutidas más precisamente. Cuando las personas distinguen la naturaleza 'siwsawi' del saber adquirido a través del lenguaje humano y la naturaleza 'ukamaw' del saber personal adquirido a través de interacciones no-linguísticas con otras cosas del mundo que llevan en si conocimientos, se usa la lógica linguística fundamental Aymara. En la sociedad Ayamara, el no-respeto de los marcadores linguísticos para indicar desde qué tipo de saber uno se expresa es percibido con suspición. Cuando los profesores de universidades no lo respetan, cuando parecen afirmar tener un conocimiento personal sobre tal tema por haber leído un libro sobre el mismo tema, los estudiantes Aymara tienden a concluir que conferencias y libros bastan cuando uno está interesado por opiniones y juicios de otras gentes, pero no representan más que 'siwsawi'; palabras pronunciadas, escuchadas y leídas, pero no experiencias vividas.

Entonces, esta manera de distinguir diferentes tipos de saber, desde sus fuentes y su fiabilidad, tiene interesantes repercusiones en el actual proceso de descolonización de la Universidad Boliviana y en la reciente creación de "universidades indígenas" que son partes integrantes de las políticas de Estado de descolonización puestas en marcha por la administración de Evo Morales. En esta presentación, llego a preguntar: si los libros y las conferencias son asuntos/cuestiones de opinión y juicios personales y que el verdadero saber sólo puede ser adquirido por la experiencia no-linguística, en interrelación con y en el mundo, el proyecto de descolonización del saber y de universidades justamente usando herramientas como libros y conferencias, ¿ no sería éste un proyecto logocéntrico de descolonización, una empresa creada y destinada a la reproducción de asimetrías epistemológicas coloniales de producción del saber? Por una parte, este trabajo subraya problemas ligados a la naturaleza 'siwsawi' del saber académico convencional (colonial) con respeto al proceso crítico de descolonización. Por otro lado, explora la naturaleza 'ukamaw' 
del saber experimental y la perspectiva para este tipo de saber, de establecer los fundamentos de una transformación epistemológicas decolonial de la Universidad Boliviana.

Fundamentalmente, este trabajo permite reflexionar sobre lo que significa adquirir conocimientos, ser sujeto y objeto de conocimiento en los Andes bolivianos hoy en día, en un contexto donde las tradiciones subalternizadas del pensamiento han tomado un rumbo importante/ urgente en las recientes dinámicas educativas y políticas y donde las distintas visiones y reivindicaciones de la verdad coexisten, fusionan y chocan.

\section{AUTEURS}

\section{ANDERS BURMAN}

University of California at Berkeley Anthropologue suédois/bolivien, titulaire d'un doctorat de l'Université de Gothenburg (2009), actuellement universitaire post-doctoral au département des études ethniques de l'université de Californie à Berkeley. 